The International Journal Of Engineering And Science (IJES)

|| Volume || 6 || Issue || 1 || Pages || PP 61-71 || 2017 ||

ISSN (e): $2319-1813$ ISSN (p): $2319-1805$

\title{
Evaluation of STBC and Convolutional Code Performance for Wireless Communication System
}

\author{
Siraj Qays Mahdi \\ Department of Computer Engineering Techniques, Electrical Engineering Technical College, Middle Technical \\ University, Baghdad, Iraq
}

\begin{abstract}
-
Under rich dissipating environment, Multiple Input Multiple Output (MIMO) scheme have better performance in term of reliability and increasing the throughput. Space Time Block Code (STBC) can reduce the Bit Error Rate (BER) with suitable data rate. In order to raise the amount of throughput more, high modulation order is used but it degrade the performance. To address this problem, a Convolutional Code (CC) can be support such system with various code rate to deal with different circumstances. This research is proposed a system with serial concatenation of STBC and CC with various modulation levels. Such system is tested with Rayleigh flat and selective fading channel by Matlab package R2015b with a list of modulation order and changing the code of each STBC and CC. The results show that such system can cover a range of Signal to Noise Ratio (SNR) from $O$ to $21 \mathrm{~dB}$ of SNR for selective fading channel and -2 to $19 \mathrm{dBfor}$ flat fading channel for a targeted BER of $10^{-4}$ with a various modulation index and code rate which lead to a flexible system to change the throughput depending on user conditions.
\end{abstract}

Keyword: MIMO, STBC, Convolutional code.

Date of Submission: 15 December 2016

\section{INTRODUCTION}

Multiple Input/ Multiple Output (MIMO) is a technology that uses multiple antennas at both the transmitter side and the receiver side. In such a way, the MIMO technology achieves high data rate for up and coming era of air interfaces like Wireless Fidelity (Wi-Fi), in view of the spatial dimension complementing Time Division Multiplexing (TDM), Frequency Division Multiplexing (FDM), and Code Division Multiplexing (CDM) multiple access technologies [1]. MIMO provides enhancedsystem due to it has numerous captivating characteristics. Where, it greatly increases the channel capacity, gives the benefit of spatial variety, increase the array gains which implies SNR gainsaccomplished by focusing energyin craved heading is expanded,be that as it may, likewise lessens the effecton ISI (inter symbol interference) and the channel fading since each signal determination depends on $\mathrm{N}$ detected outcomes[2]. The MIMO system for multiple antennas permits to perform pre-coding spatial multiplexing in addition to diversity coding. The network capacity is increased from using the spatial multiplexing method by parting a high rate signal into various lower rate streams and transmitting them through various receiving antennas. But a high error rate is adopted within the transmitting system [3].

As a way to enhance the system performance, the space time block code is utilized to enhance the data transmission reliability of wireless communicationby utilizing numerous transmits antennas. For this system, which deals with space time block code are more reliable by reducing the error rate as composed to the Spatial multiplexing method[4].

Convolutional code is one oferror-correcting code type that generates parity signs by method of the sliding use of a Boolean polynomial functionof an information stream. The sliding application speaks to the "convolution" of the encoder over the information, which proffers ascend to the term 'convolutional coding. The sliding way of the convolutional codes encourages trellis decoding using a time invariant trellis.Hence, convolutional code has many attributes;convolutional codes are described by the base code rate and the memory of the encoder $[n, k$, $K]$. Convolutional codes are continuous. Where, it is performed on blocks of data. Moreover, Convolutional codes are utilized broadly to accomplish reliable data transfer from various applications, for example, computerized video, radio, portable correspondences and satellite communication [5].

Furthermore, the performance of the system can be improvement through concatenation the space time block code by convolutional code. The concatenation is in diversity order by the presentation of convolutional codes into space-time block coded system. Where, the general analytical expression of the error correction of space time block code is determined.

A number of researchers were interested in field of MIMO wireless communication to design STBC with variable number ofcoding.In [6], they have been presented the improvement of BER performance of 64PSK 
STBC utilizing turbo code. Where, two main parts are used in STBC decoder in addition to channel estimator and maximum ratio combiner. The simulation resultsusing (matlab 2010) shows that STBC rate is 0.5 for STBC3 and modulation order is small of 2PSK.

In [7], they have been viewed as the issue of space-frequency code designs for frequency- selection multiple MIMO Orthogonal Frequency Division Multiplexing (OFDM) Modulation. The codes are built utilizing basic mapping from space- time codes to space- frequency block.MIMOtechnique is presented in [8] to be essential for BWM system (IEEE 802.16e) which is prominently known as mobile WiMAX system. They achieve a BER which improved using MIMO technique.In [9], they proposed system consolidates between two types of diversity structures, frequency diversity (denoted by OFDM) and antenna diversity (represented by STBC scheme). Where, three techniques were used for decreasing the Peak to Average Power Ratio (PAR). The result demonstrated that BER has reached to satisfy level with low SNR.

This paper concerned to design STBC system supported by convolutional code with variable code rate and multi-level of data mapping to optimize their parameters for both STBC and convolutional code to achieve vary low BER and suitable bit rate.

\section{SPACE TIME BLOCK CODE (STBC)}

MIMO system made out of three primary components, the Transmitter $(T x)$, the Receiver $(R x)$ and the Channel $(\breve{M})$ as shown in Fig. (1).

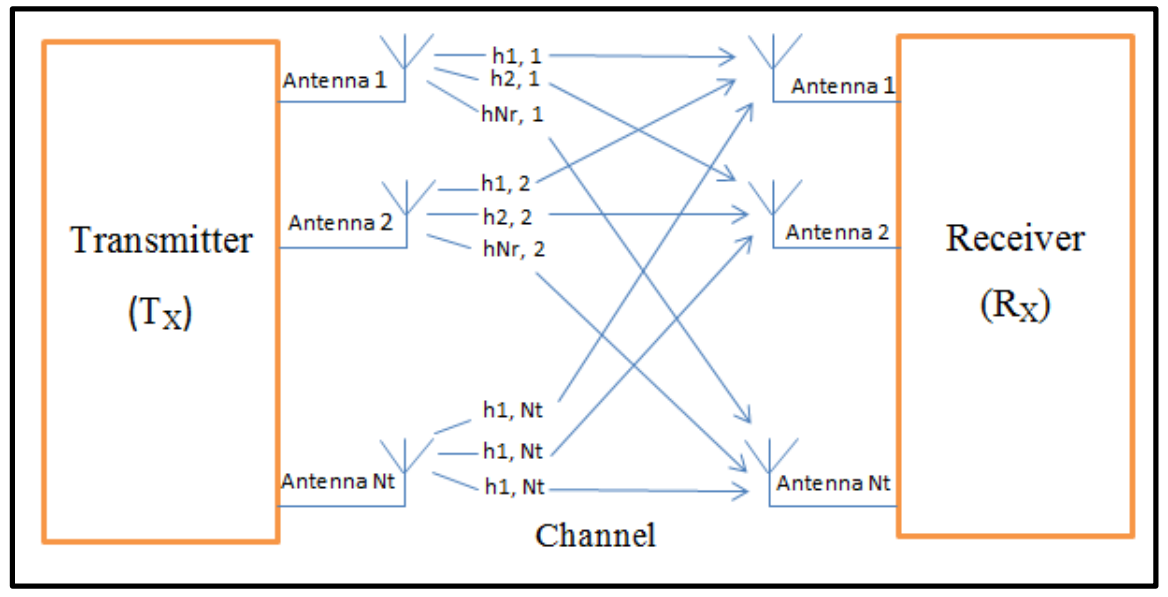

Fig. 1:Block Diagram of MIMO System.

The channel of MIMO system is represented by a matrix called channel matrix.

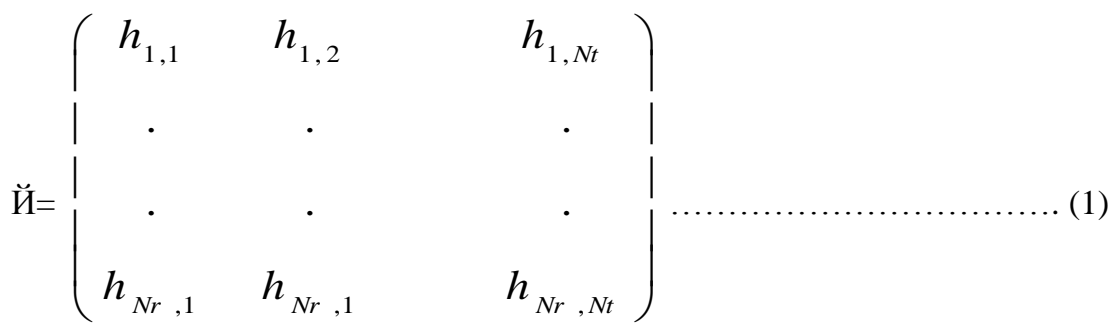

Where, $N_{t}$ and $N_{r}$ are the number of antennas at the transmitter side and receiver side respectively. Each input $h_{i, j}$ signifies the attenuation and phase shift between the $j t h$ transmitter side and $i_{t h}$ receiver side [3].

$h=\alpha e^{j \theta} \delta(t+\tau)$

However, STBC is a technique utilized as a part of MIMO wireless communication system using to transmit numerous duplicates of information stream over various numbers of antennas and to take advantage of the different received versions of the information to enhance the unwavering quality of data transfer.Indeed, the transmitted signal composed to scattering, reflection, refraction and so on through the environment in addition to further thermal noise is corrupted at the receiver that mean many of received copies better than others. Therefore, the receiver decoder combines all the copiers in an ideal approach to concentrate much data from the 
all the received signals [10].The row of the STBC channel matrix represents a time slot and each column represents one antenna's transmissions after some time as follow:

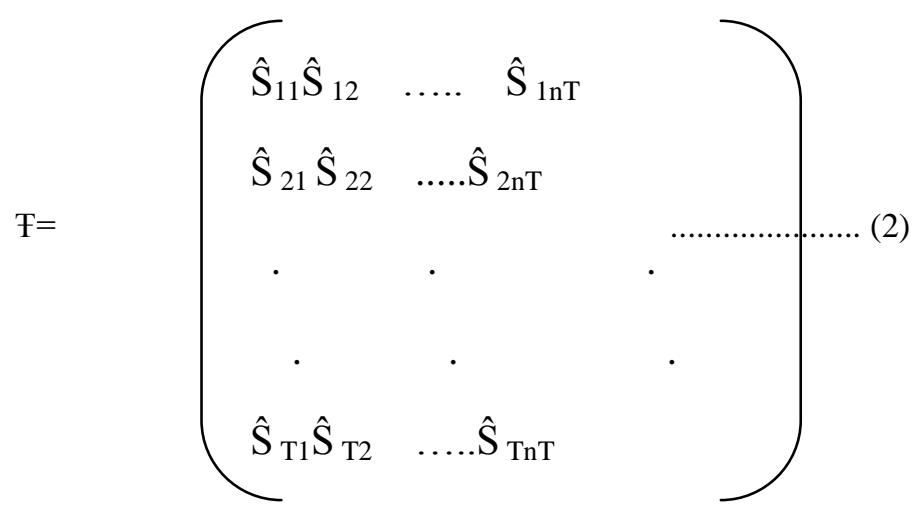

Where $F$ is the time slot and the modulated symbol is $\hat{S}_{i j}$ to be transmitted at time slot $i$ from antenna $j$. there are to inform $F$ time slots [11]. The code rate of STBC measure how much time slot is transmitted and can be calculated as follow:

r $=\frac{\mathrm{K}}{\mathrm{F}}$. .

Where, $K$ is blocking code.

Thegain array of MIMO system is increased as a result of the relation between the number of transmitter or receiver antennas or both with SNR. To show the relation between the channel capacityand the number of receive and transmit antennas. It must start with Shannon equation [12].

$\mathrm{C}=\mathrm{M} \log _{2}(1+\mathrm{SNR}) \mathrm{Bits} / \mathrm{sec} / \mathrm{unit}$ area

Where, $M$ is the channel bandwidth.Moreover, increasing the number of transmit and receive antennas lead up to gradual increase of channel capacity. This prompt gave extra capacity with no expansion in bandwidth.

Reasonably, various data streams can be gotten from the MIMO system to be transmitted at same frequency. So,this leads to increase the bandwidth efficiency. The MIMO system capacity $\beta_{M I M O}$ is appeared to be [13]:

$\beta_{\mathrm{MIMO}}=\log _{2}\left(\operatorname{det}\left(\mathrm{I}+\frac{\mathrm{SNR}}{\mathrm{n}_{\mathrm{T}}} \breve{и ̆}^{+}\right)\right)$

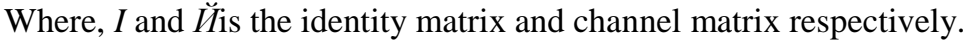

The code rate $r$ with the capacity of corresponding STBC channel is known by [14]:

$\ell_{\text {STBC }}=M \operatorname{Ṃ́}^{\prime} \log _{2}\left(\operatorname{det}\left(I+\frac{\text { SNR }}{\mathrm{Rn}_{\mathrm{T}}} \breve{и ̆}^{+}\right)\right)$

\section{CONVOLUTIONAL CODE}

Convolutional codes belong to the Forward Error Correcting (FEC) scheme is a process of channel coding. Convolutional code has three parameters $(\tilde{N}, \dot{K}, L)$ where $\tilde{N}$ is the number of outputs at the encoder, $\dot{K}^{\prime}$ is the number of bits we present at the input of the encoder at a time, $M$ is the memory length and $L$ is the memory length plus the input $(L=M+1)$ as shown in Fig.2.

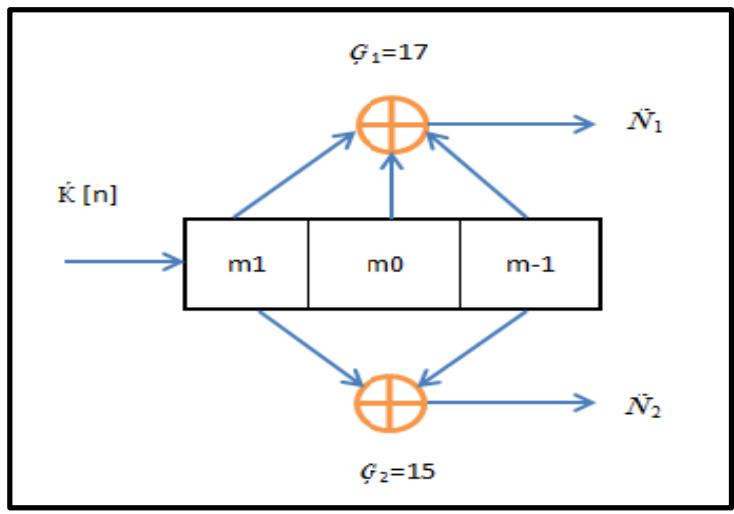

Fig. 2: convolutional encoder. 
The proportion of input bit to the output bit is denoted the coding rate of the convolutional codes i.e. $\dot{I}^{\prime}=\dot{K}^{\prime} / \tilde{N}$. Hence, it chooses the substance of data and the overhead of coding. The memory is stored the previous state and the input message of the encoder todetermine the encoder output [5].

The input bits $K^{\prime}[n]$ goes through the encoder. At that point, the output bits of the encoder are computed throughutilizing modulo-2 additions, which resembles XORprocess of the current state and the content of the previous state of the register at the encoder. Where,the encoder comprises two memories to handle the incoming bits. At long last, the output bits $V_{l}$ and $V_{2}$ aremultiplexingto produce the code word.

The code rate $\dot{I}$ and the constraint length $K^{\prime}$ determine the performance of the convolutional code. Hence, More powerful code and more coding gain are contributes from the longer constraint length. However, more complex decoder and more time are required for the decoding. Correspondingly, more effective code is provided from smaller coding rate because of further redundancy butless data transfer capacity effectiveness comparing to higher coding rate. The convolutional code can be depicted by the generator sequence $G^{(1)}, G^{(2)}, G^{(n)}$, which is likewise called the encoder impulse response [15].

Every polynomial has a less grade than or equal to $(\dot{K} \times k-1)$ that depicts the relationship of the shift registers to the related mudulo-2 adder. Thus, two mudulo-2 adders, the generator polynomial $G^{l}(Y)$ and $G^{2}(Y)$ are given by:

$\mathrm{G}_{1}(\mathrm{Y})=\mathrm{G}_{0}{ }^{(1)}+\mathrm{G}_{1}{ }^{(1)} \cdot \mathrm{Y}+\mathrm{G}_{2}{ }^{(1)} \cdot \mathrm{Y}_{2}+\mathrm{G}_{3}{ }^{(1)} \cdot \mathrm{Y}_{3}$

In $G_{0}{ }^{(1)}=1,1$ clarify the modulo- 2 adder is associated to the shift registers. Additionally, in ${ }_{1}{ }^{(1)}=0,0$ represents the modulo- 2 adder which is not connected to the shift registers.

In [14], and return to Fig. (2).

$\mathrm{G}_{2}{ }^{(1)}=1$ and $\mathrm{G}_{3}{ }^{(1)}=1$ are obtainable. $\mathrm{G}_{1}(\mathrm{Y})=1+\mathrm{Y}_{1}+\mathrm{Y}_{2}+\mathrm{Y}_{3}$

Similarly,

$\mathrm{G}_{2}(\mathrm{Y})=\mathrm{G}_{0}{ }^{(2)}+\mathrm{G}_{1}{ }^{(2)} \cdot \mathrm{Y}+\mathrm{G}_{2}{ }^{(2)} \cdot \mathrm{Y}_{2}+\mathrm{G}_{3}{ }^{(2)} \cdot \mathrm{Y}_{3}=1+\mathrm{Y}+\mathrm{Y}_{3}$

From the above polynomial equations, the polynomial sequence which obtained is:

$G_{1}=(1,1,1)$

$\mathrm{G}_{2}=(1,0,1)$

Also, the output sequence is given by,

" $(\mathrm{Y})=\mathrm{u}(\mathrm{Y}) \mathrm{G}_{1}(\mathrm{Y})$ multiplexed with $\mathrm{u}(\mathrm{Y}) G_{2}(\mathrm{Y})$.

In this research has been adopted Viterbi algorithm to achieve an efficient decoder. Moreover, Concatenated Codesare formed by combination of space time block code with convolutional code to increase the potential of such scheme.

\section{SYSTEM MODEL.}

The system model which adopted in this research is as shown in Fig. (3). This section will explain briefly the function of each block to clear out the overall system.
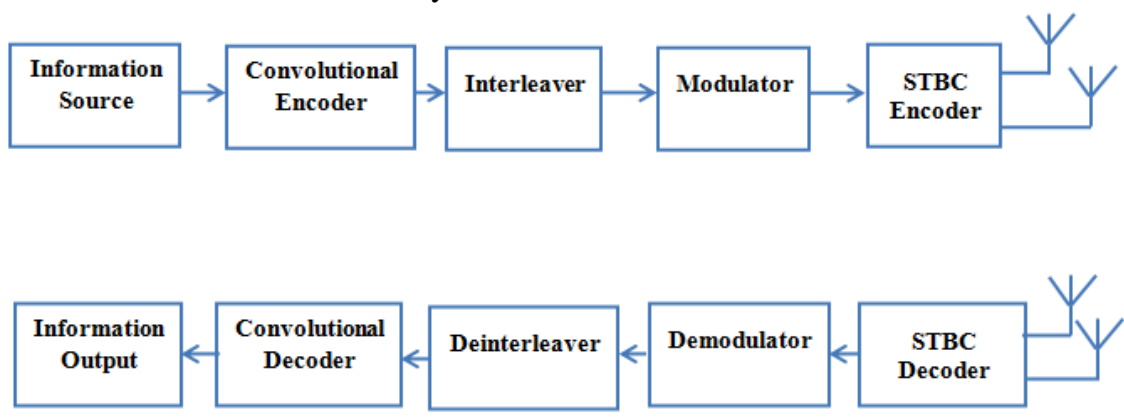

Fig. 3:Block Diagram of the System Model.

The information source produces the data message in a form of stream bits where the probability of ones equal to 0.5 . The data is fed to convolutional code to produce coded data. In this research a variable code rate has been adopted using puncturing technique which eliminates some output bits to increase the data rate. The code rate isvaried depending on condition of the channel. In worse case of channel, low code rate will be used to address the high error rate and vice versa.

To increase the convolutional code efficiency, it has been used the block interleaver to separate the burst error and improve the decoder performance. Thecoded word is fed to modulator to be mapped it into a form of symbol that contain a number of bits depending on the level of modulation. In this research, it has been adopted QAM, 8QAM and 16QAM, 32QAM and 64 QAM to form a symbol with 2, 3, 4, 5 and 6 bits respectively. The STBC encoder is encoded the serial stream symbol to be transmitted over multiple antenna. In this research, it has been used $4 \times 2$ and $4 \times 4$ antennas with a code rate of 1/2, 2/3and 3/4 to improve the system 
performance.Finally, the channel used in such system is flat and selective fading. For flat fading, the parameter used for simulation is listed in table1. Also, the parameters of selective fading are listed in table 2 . The function of rest received system is the inverse of the corresponding block at the transmitter side.

Table (1):Flat fading Parameters

\begin{tabular}{|c|c|}
\hline Parameter Type & Type or Value \\
\hline Channel & "Rayleigh" \\
\hline "InputSamplePeriod" & "1.0000e-07" \\
\hline "DopplerSpectrum" & $100,0,0$ \\
\hline MaxDopplerShift, Path Delays, Avg. Path Gain dB & $1,0,0$ \\
\hline $\begin{array}{c}\text { NormalizePathGains, Store History, Store Path } \\
\text { Gains }\end{array}$ & $-0.3397-0.2994 \mathrm{i}$ \\
\hline "PathGains" & 720000 \\
\hline
\end{tabular}

Table (2): Selective fading Parameters.

\begin{tabular}{|c|c|}
\hline Parameter Type & Type or Value \\
\hline Channel & "Rayleigh" \\
\hline "InputSamplePeriod" & "1.0000e-07" \\
\hline "DopplerSpectrum" & 100,01 doppler.jakes" \\
\hline Max. DopplerShift, Path Delays, Avg. Path Gain dB & $1,0,0$ \\
\hline $\begin{array}{c}\text { NormalizePathGains, Store History, Store Path } \\
\text { Gains }\end{array}$ & {$[-0.1072+0.0592 \mathrm{i}-0.4446-0.1738 \mathrm{i}]$} \\
\hline "PathGains" & 720000 \\
\hline
\end{tabular}

\section{SIMULATION AND RESULTS}

Simulations have been implemented to analyze the performance ofthesystem model which includesthe STBC with convolutional code. The tool that uses to measure such performance is BER versus SNR. To show the potential for such system, it has been uses various types of modulation index, channels and code rate of each space time and convolutional code in addition to number of transmitted and received antennas. Table (3) listed the values and types of all elements used.

Fig.4 illustrates the results of using 4x2 antennas, flat fading, 1/2 code rate for each space time block code and convolutional code or overall code rate is $1 / 4$.

Table (3): A list of all elementsused in the simulation.

\begin{tabular}{|c|c|c|c|}
\hline \multirow{2}{*}{ No. of antenna } & Type of channel & Overall Code Rate & Modulation index \\
\hline $4 \times 2$ & Rayleigh, AWGN & $1 / 4$ & QAM, 8QAM, 16QAM, 64QAM \\
\hline $4 \times 2$ & Rayleigh, AWGN & $1 / 3$ & QAM, 8QAM, 16QAM, 64QAM \\
\hline $4 \times 2$ & Rayleigh, AWGN & $3 / 8$ & QAM, 8QAM, 16QAM, 64 QAM \\
\hline $4 \times 2$ & Rayleigh, AWGN & $1 / 2$ & QAM, 8 QAM, 16 QAM, 64 QAM \\
\hline $4 \times 4$ & Rayleigh, AWGN & $1 / 4$ & QAM, 8 QAM, 16 QAM, 64 QAM \\
\hline $4 \times 4$ & Rayleigh, AWGN & $1 / 3$ & QAM, 8 QAM, 16 QAM, 64 QAM \\
\hline $4 \times 4$ & Rayleigh, AWGN & $3 / 8$ & QAM, 8 QAM, 16 QAM, 64 QAM \\
\hline
\end{tabular}

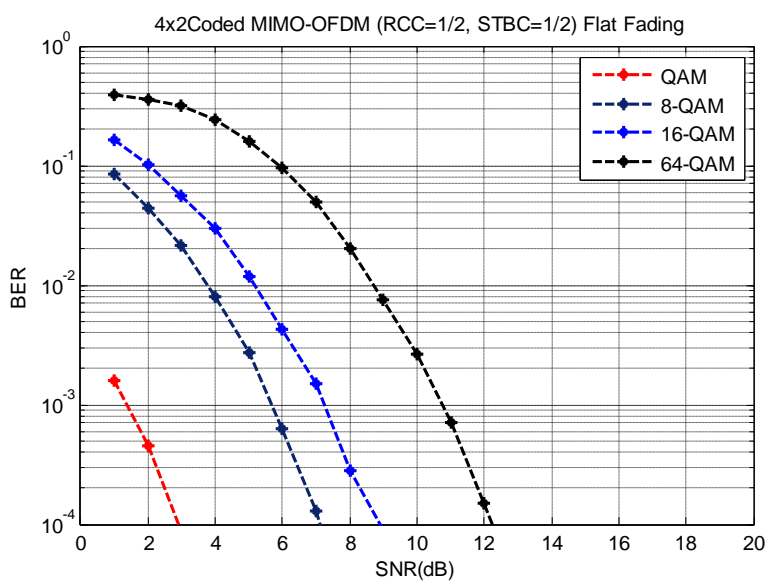

Fig. 4: Flat fading (4x2) transmitter-receiver with overall code rate $1 / 4$ 
It has been clear out that better performance is obtained with QAM, it needs only $3 \mathrm{~dB}$ SNR to achieve $10^{-4}$ BER while 64 QAM needs about $12 \mathrm{~dB}$ SNR to reach the BER, but taking into account that the bit rate in 64 QAM is three times bit rate than QAM, while 8 QAM and 16 QAM are in-between of them.

Now, the same scheme of previous case except the channel is changed to frequency selective fading instead of flat fading, the results is shown in Fig. 5.

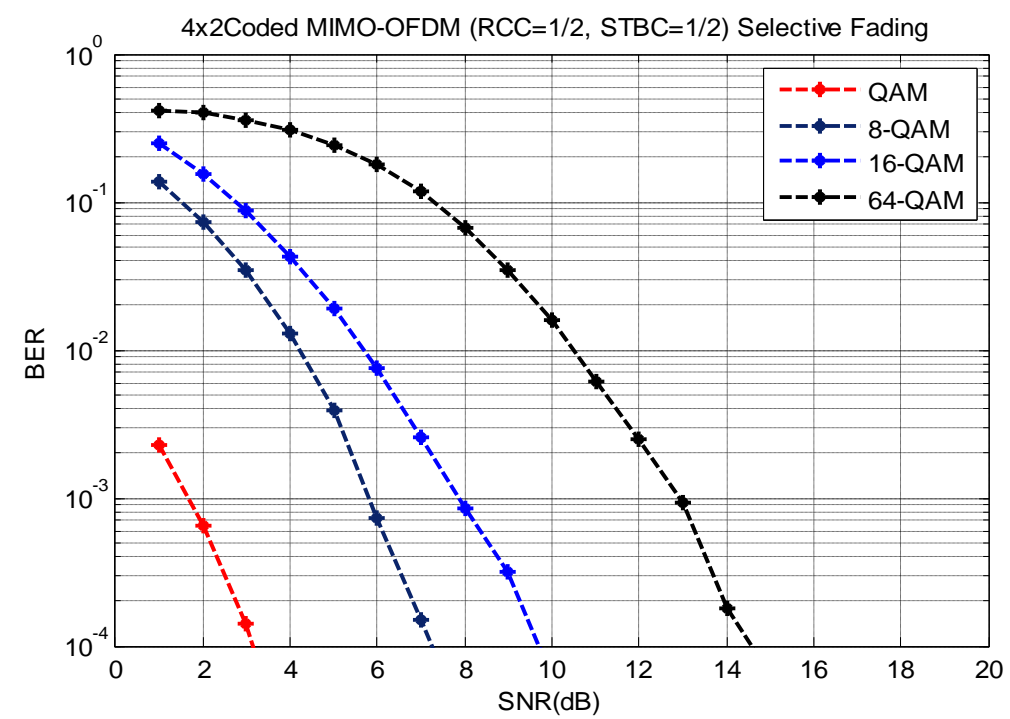

Fig.5: Selective fading $(4 \times 2)$ transmitter-receiver with code rate $1 / 4$.

The performance of the system with selective fading is the same as in flat fading but increasing the SNR slightly for each case because of hug noise that subjected tothe signal, but the system is appeared as a robust in worst environments.

It has been repeated same simulations with other code rate $(1 / 2 \mathrm{STBC}$ and $2 / 3 \mathrm{CC})$ or $1 / 3$ overall code rate. The results are shown in Fig. 6.

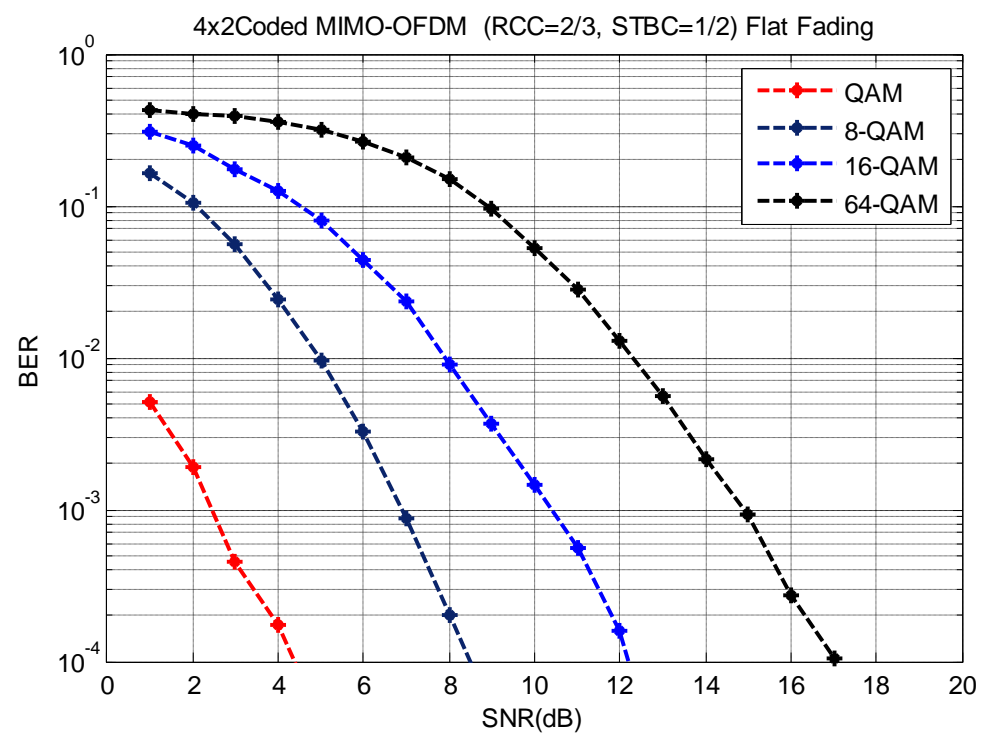

Fig. 6:Flat fading $(4 \times 2)$ transmitter-receiver with code rate $1 / 3$.

In this case, the 64 QAM needs $17 \mathrm{~dB}$ of SNR to achieve $10^{-4}$ BER while QAM needs only $4.5 \mathrm{~dB}$ of SNR to approach same BER. But note that the overall code rate here is $1 / 3$, that means it can be increase the throughput of the system by a ratio of $4 / 3$ in return to previous case which used overall code rate of $1 / 4$. The results of selective fading channel of such case are shown in Fig. 7. It is clear out that the performance needs more SNR because the environment of selective fading and increasing the code rate value. 


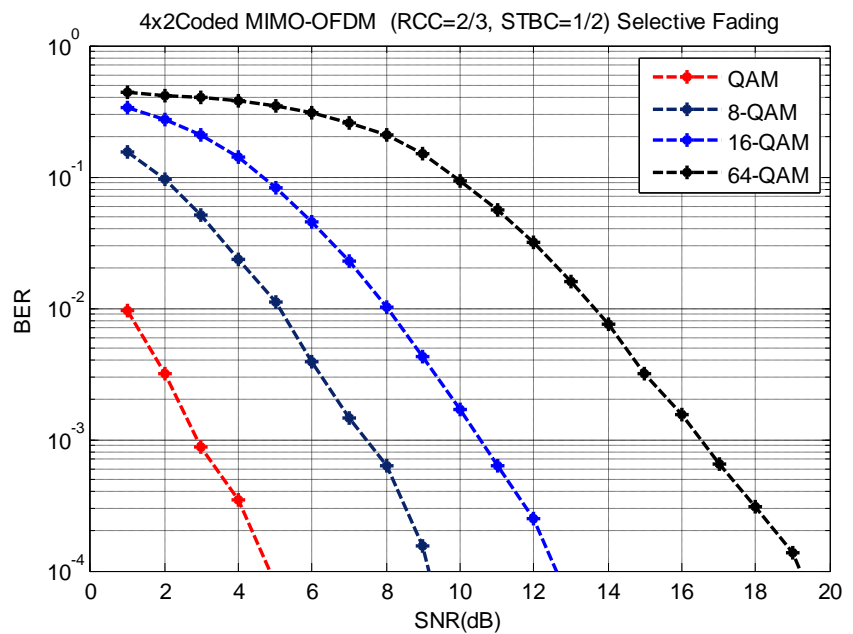

Fig. 7:Selective fading $(4 \times 2)$ transmitter-receiver with code rate 1/3.

Fig. 8 shows the case of overall code rate (3/8) using flat fading frequency. Itshows that 64 QAM approached to $10^{-4}$ BER with $17 \mathrm{~dB}$ SNR while QAM needs 3.5 SNR to reach the targeted BER $\left(10^{-4}\right)$. The results of selective fading channel used for this case is shown in Fig. 9.

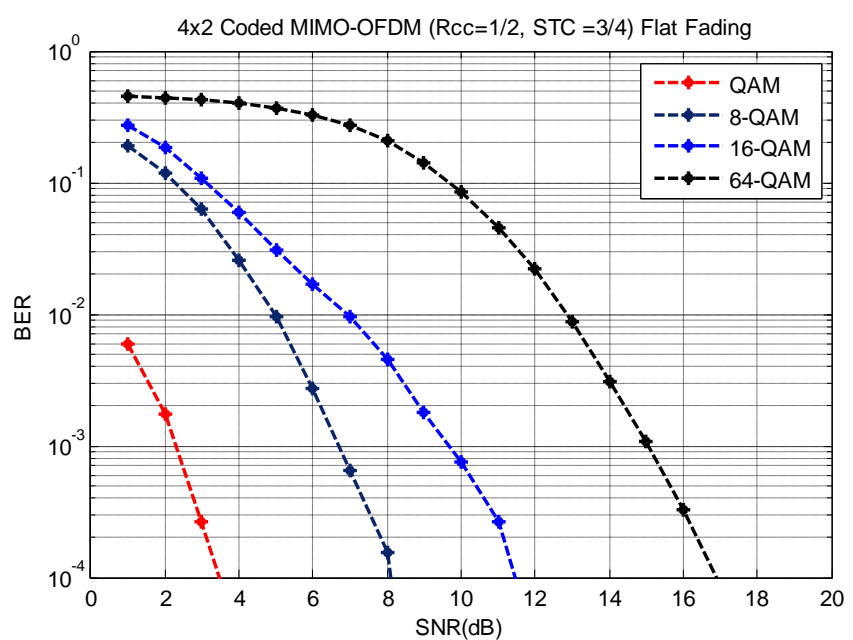

Fig.8: Flat fading $(4 \times 2)$ transmitter-receiver with overall code rate $3 / 8$.

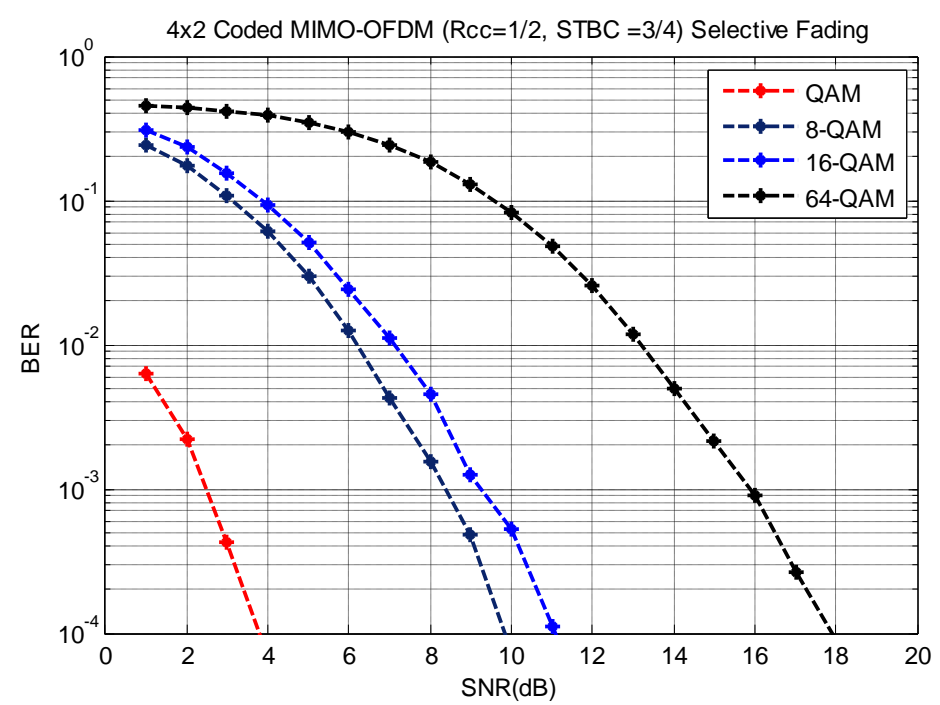

Fig.9: Selective fading $(4 \times 2)$ transmitter-receiver with code rate $3 / 8$. 
Also, $1 / 2$ overall code rate is used for same previous system with flat and selective fading channels. The results are illustrated in Fig. 10 and Fig. 11 for flat and selective fading respectively. Furthermore, it has been repeated all previous simulations but using $4 \times 4$ antennas. Figures $(12,13,14,15,16$ and 17) are clear out the results. The amounts of SNR needed for each all previous cases to approach the targeted 10-4 BER are summarized in table (4).

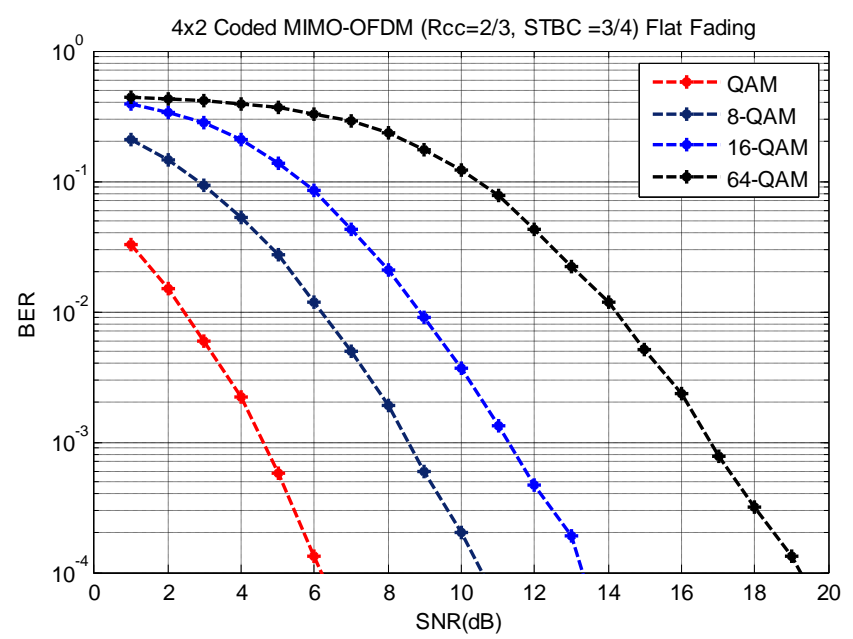

Fig.10: Flat fading $(4 \times 2)$ transmitter-receiver with code rate $1 / 2$.

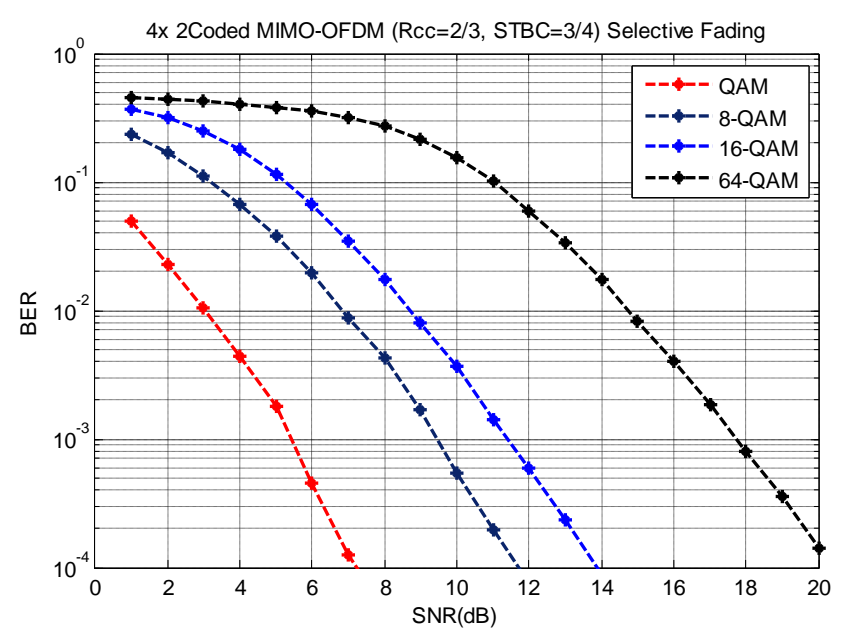

Fig.11: Selective fading (4x2) transmitter-receiver with code rate 1/2.

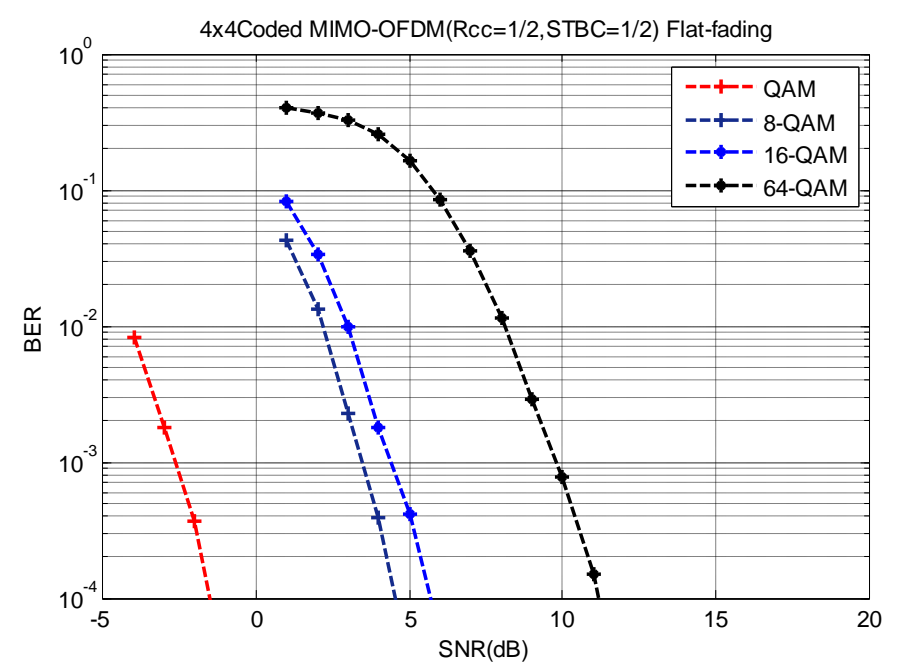

Fig.12: Flat fading $(4 \times 4)$ transmitter-receiver with code rate $1 / 4$. 


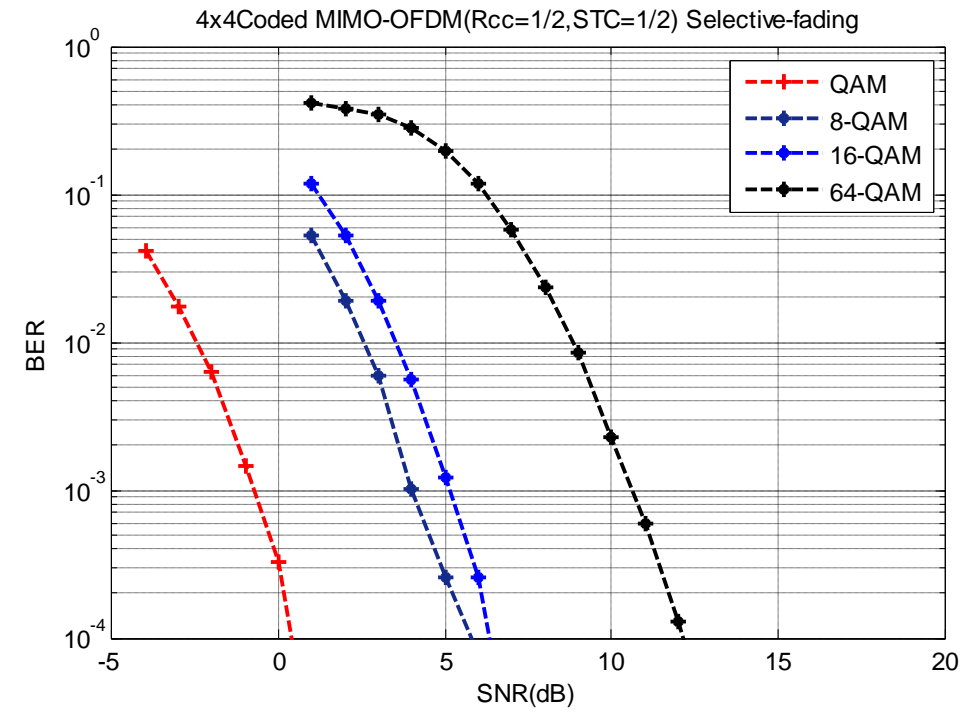

Fig.13: Selective fading $(4 \times 4)$ transmitter-receiver with code rate $1 / 4$.

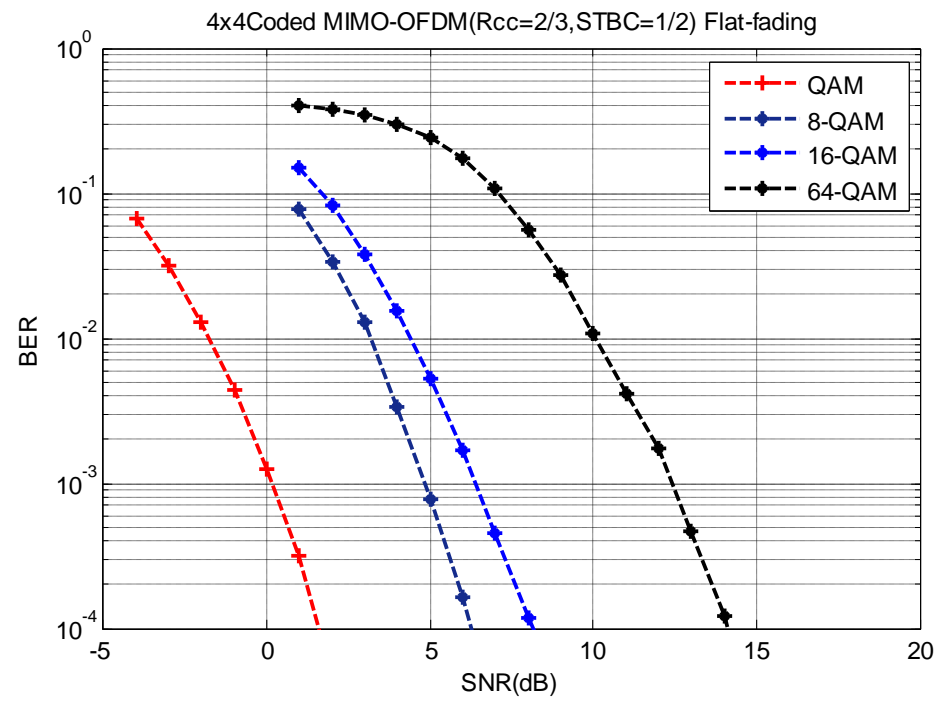

Fig.14: Flat fading $(4 \times 4)$ transmitter-receiver with code rate $1 / 3$.

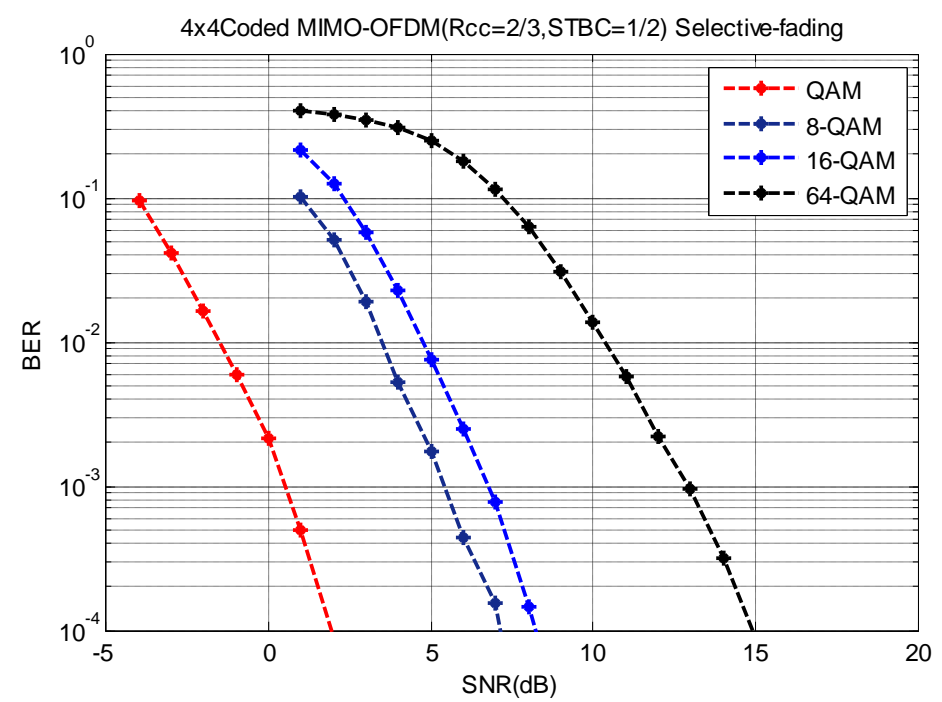

Fig.15: Selective fading $(4 \times 2)$ transmitter-receiver with code rate 1/3. 


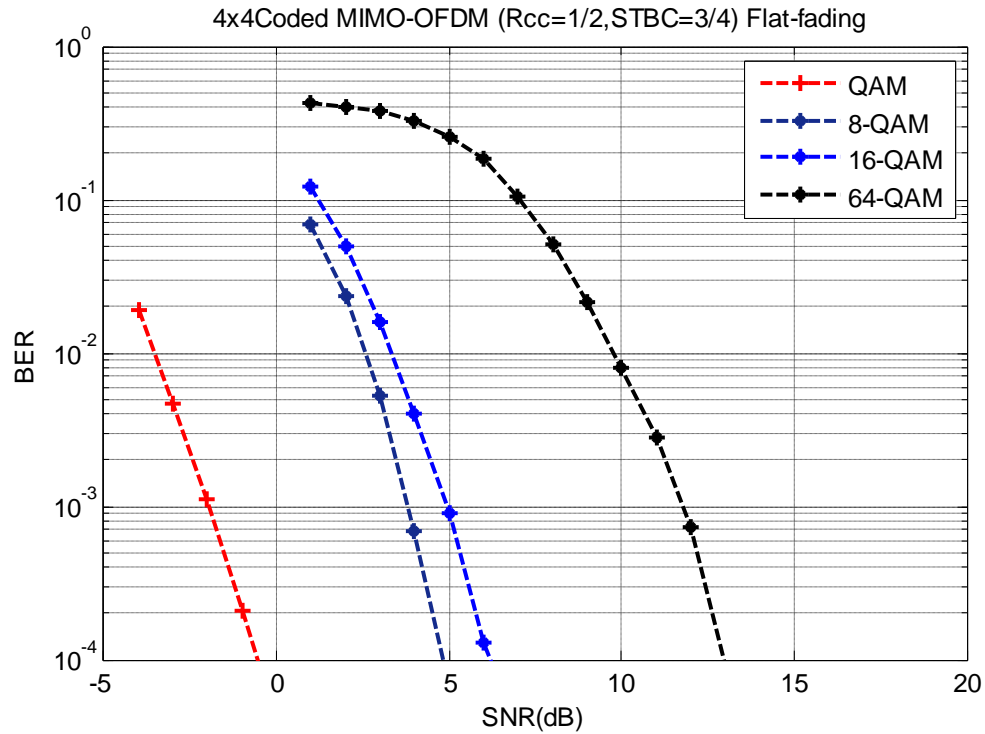

Fig.16: Flat fading $(4 \times 4)$ transmitter-receiver with code rate $3 / 8$.

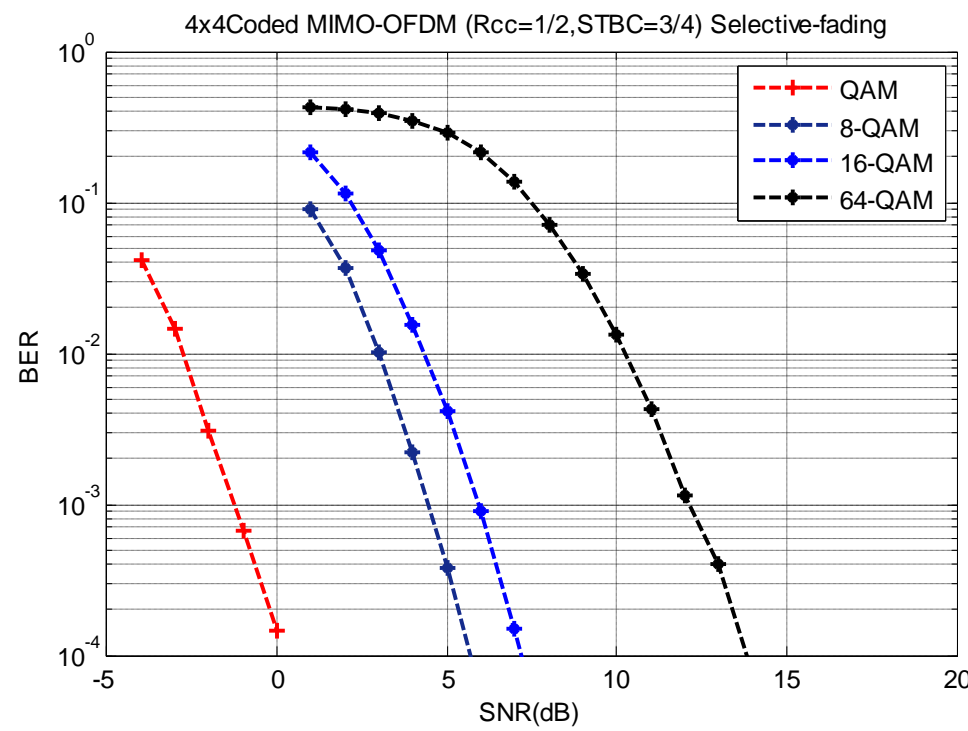

Fig.17: Selective fading $(4 \times 4)$ transmitter-receiver with code rate $3 / 8$.

Table (4): the results of all simulations with targeted BER $\left(10^{-4}\right)$.

\begin{tabular}{|c|c|c|c|c|c|}
\hline \multirow[b]{2}{*}{$\begin{array}{l}\text { No. of } \\
\text { antenna }\end{array}$} & \multirow{2}{*}{$\begin{array}{l}\text { Type of } \\
\text { channel }\end{array}$} & \multirow{2}{*}{$\begin{array}{l}\text { Overall Code } \\
\text { Rate }\end{array}$} & \multirow[b]{2}{*}{$\begin{array}{l}\text { Modulation } \\
\text { index }\end{array}$} & \multicolumn{2}{|c|}{ SNR } \\
\hline & & & & $\begin{array}{c}\text { Flat } \\
\text { Fading }\end{array}$ & $\begin{array}{l}\text { Selective } \\
\text { Fading }\end{array}$ \\
\hline \multirow{4}{*}{$4 \times 2$} & \multirow{4}{*}{$\begin{array}{l}\text { Rayleigh, } \\
\text { AWGN }\end{array}$} & $1 / 4$ & QAM & 3 & 3.2 \\
\hline & & $1 / 4$ & 8 QAM & 7 & 7.5 \\
\hline & & $1 / 4$ & 16 QAM, & 9 & 9.5 \\
\hline & & $1 / 4$ & 64 QAM & 12 & 14.5 \\
\hline \multirow{4}{*}{$4 \times 2$} & \multirow{4}{*}{$\begin{array}{l}\text { Rayleigh, } \\
\text { AWGN }\end{array}$} & $1 / 3$ & QAM & 4.5 & 5 \\
\hline & & $1 / 3$ & $8 \mathrm{QAM}$ & 8.5 & 9 \\
\hline & & $1 / 3$ & 16 QAM & 12 & 12.5 \\
\hline & & $1 / 3$ & 64 QAM & 17 & 19 \\
\hline \multirow{4}{*}{$4 \times 2$} & \multirow{4}{*}{$\begin{array}{l}\text { Rayleigh, } \\
\text { AWGN }\end{array}$} & $3 / 8$ & QAM & 3.5 & 4 \\
\hline & & $3 / 8$ & 8 QAM, & 8 & 10 \\
\hline & & $3 / 8$ & 16 QAM, & 11.5 & 11.5 \\
\hline & & $3 / 8$ & 64 QAM & 17 & 18 \\
\hline \multirow{4}{*}{$4 \times 2$} & \multirow{4}{*}{$\begin{array}{l}\text { Rayleigh, } \\
\text { AWGN }\end{array}$} & $1 / 2$ & QAM & 6 & 7 \\
\hline & & $1 / 2$ & 8 QAM & 10.5 & 11.5 \\
\hline & & $1 / 2$ & 16 QAM & 13 & 14 \\
\hline & & $1 / 2$ & 64 QAM & 19 & 21 \\
\hline
\end{tabular}




\begin{tabular}{|c|c|c|c|c|c|}
\hline \multirow{4}{*}{$4 \times 4$} & \multirow{4}{*}{$\begin{array}{l}\text { Rayleigh, } \\
\text { AWGN }\end{array}$} & $1 / 4$ & QAM & -2 & 1 \\
\hline & & $1 / 4$ & 8 QAM & 4.5 & 6 \\
\hline & & $1 / 4$ & 16 QAM & 6 & 7 \\
\hline & & $1 / 4$ & 64 QAM & 11.5 & 12 \\
\hline \multirow{4}{*}{$4 \times 4$} & \multirow{4}{*}{$\begin{array}{c}\text { Rayleigh, } \\
\text { AWGN }\end{array}$} & $1 / 3$ & QAM & 2 & 3 \\
\hline & & $1 / 3$ & 8 QAM, & 6.5 & 7 \\
\hline & & $1 / 3$ & 16 QAM & 8 & 8.5 \\
\hline & & $1 / 3$ & 64 QAM & 14 & 15 \\
\hline \multirow{4}{*}{$4 \times 4$} & \multirow{4}{*}{$\begin{array}{l}\text { Rayleigh, } \\
\text { AWGN }\end{array}$} & $3 / 8$ & QAM & -1 & 0 \\
\hline & & $3 / 8$ & 8 QAM, & 5 & 5.5 \\
\hline & & $3 / 8$ & 16 QAM, & 6.5 & 7.5 \\
\hline & & $3 / 8$ & 64 QAM & 13 & 13.5 \\
\hline
\end{tabular}

\section{CONCLUSIONS}

In this research, it has been proposed a system consisted of STBC assisted by convolutional code to achieve a reliable received data for wireless communication. Various code rate for each convolutional code and STBC in addition to varying modulation index. The results show that this system can be approach very low BER at suitable SNR. For example, the system with QAM level of modulation, overall code rate of $3 / 8$ and uses $4 \times 4$ antennas can achieves $10^{-4}$ BER at $0 \mathrm{~dB}$ of SNR in the environments of selective fading channel and $-2 \mathrm{~dB}$ for flat fading but 1/4 overall code rate. The proposed system can cover a range of SNR from 0 to $21 \mathrm{~dB}$ at a targeted BER of $10^{-4}$ for Rayleigh selective fading and -2 to $19 \mathrm{~dB}$ with a several code rate and modulation index. So this system is useful for modern communications for being flexible in changing the data rate in different circumstances.

For future research, it can be design a dynamic system based on the results achieved by this research to increase the throughput by putting a suitable code rate and constellation level for each received SNR.

\section{REFERENCES}

[1]. Varsha Rao Bolar," Performance Analysis Of Space - Time Block Coded Multiple Antenna Systems", Master Thesis, The University of Texas, Master Of Science In Electrical Engineering, December 2009.

[2]. Jing Xiao, "Analysis of OFDM MIMO", Master Thesis in Electrical Engineering, Faculty of San Diego State University. Summer 2010.

[3]. Rajeev Singh, "MIMO System Using Space-Time Block Code with Digital Modulation Techniques", International Journal of Electrical and Electronics Research (IJEER), Vol. 1, Issue 1, pp: (26-31), Month: October-December 2013.

[4]. Keyur U. Chauhan, Bhargav B. Patel and Sagar B. Patel, "Performance Evaluation of Space Time Block Codes Using Bit Error Rate in MIMO Systems", International Journal of Emerging Technology and Advanced Engineering, ISSN 2250-2459, ISO 9001:2008 Certified Journal, Volume 5, Issue 1, January 2015.

[5]. Bhaskar Nandy, "Analysis of Convolutional Encoder System", International Journal of Current Engineering and Technology, EISSN 2277 - 4106, P-ISSN 2347 - 5161, Vol.6, No.2 (April 2016).

[6]. Samir Abd AlCathem Khother, "Improve the BER Performance of 64PSK, 4×2 STBC using $(8,4)$ exHamming Turbo Product Code", Journal of Babylon University/Engineering Sciences/ No. (5)/ Vol. (21): 2013.

[7]. Michael L. McCloud, Matthias Brehler, and Mahesh K. Varanasi, "Signal Design and Convolutional Coding for Noncoherent Space-Time Communication on the Block-Rayleigh-Fading Channel", IEEE Transactions on Information Theory, Vol. 48, No. 5, May 2002.

[8]. Nepal Narayan and Mohammed Tarique, "Performance evaluation of MIMO based WiMAX system", Cyber Journals: Multidisciplinary Journals in Science and Technology, Journal of Selected Areas in Telecommunications (JSAT), July Edition, 2011 .

[9]. V.RamaKrishna1, P.SrinivasaRao, "Performance Analysis of MIMO using Space Time Block Coded Spatial Domain", International Journal of Application or Innovation in Engineering \& Management (IJAIEM), Volume 2, Issue 8, August 2013.

[10]. Krishna K. Gupta, and Leela K. Balivada, "Performance Analysis of Orthogonal Space-Time Block Code over Rayleigh Fading Channels for Various Modulation Techniques", International Journal Of Computer Applications (0975 - 8887), Volume 77 No.6, September 2013.

[11]. Subhashini Dhiman, "Performance Analysis Of Time Reversal Space Time Block Codes In Nakagami Fading Channel", Master Thesis, Thapar University, Electronics and Communication Engineering Department, India,July-2014.

[12]. S. Sandhu and A. Paulraj, "Space-Time Block Codes: A Capacity Perspective", IEEE Commun. Lett., vol. 4, no. 12, pp. 384.386, Dec. 2000 .

[13]. C. C. Chiau "Study of the Diversity Antenna Array for the MIMO Wireless Communication System" Ph.D. thesis, Department of Electronic Engineering Queen Mary, University of London, April 2006.

[14]. A. S.Hiwale and Dr. A. A. Ghatol, "Capacity and Performance Analysis of Space-Time Block Codes in Rayleigh Fading Channels", wseas Transactions on Communications. Issue 12, Volume 6, pp 861- 866, December 2007.

[15]. Sundar Rupakheti, "Burst Error Correcting Convolutional Codes For Broadband Wireless Transmission Systems", Master Thesis, School of Engineering and IT, Charles Darwin University, October 2013. 